\title{
Spatially-resolved high-spectral resolution observations of the red supergiant Betelgeuse
}

\author{
K. Ohnaka ${ }^{1}$
}

\author{
${ }^{1}$ Max-Planck-Institut für Radioastronomie, Auf dem Hügel 69, 53121 Bonn, Germany \\ email: kohnaka@mpifr-bonn.mpg.de
}

Red supergiants (RSGs) experience slow, intensive mass loss up to $10^{-4} M_{\odot} \mathrm{yr}^{-1}$. Despite its importance not only in stellar evolution but also in the chemical enrichment of the interstellar matter, the mass loss mechanism in RSGs is not well understood. A better understanding of the outer atmosphere of RSGs is a key to unraveling the mass-loss mechanism in these stars. High spatial resolution observations in IR molecular lines are very effective for probing the physical properties of the inhomogeneous outer atmosphere. We observed the prototypical RSG Betelgeuse (M1-2Ia-Ibe) in the CO first overtone lines with the spectro-interferometric instrument AMBER at the ESO's Very Large Telescope Interferometer (VLTI) using baselines of 16,32 , and $48 \mathrm{~m}$. Details of the observations and the modeling are described in Ohnaka et al. (2009). The high-spectral $(R=4800-12000)$ and high-spatial resolution (9 mas) provided with AMBER allowed us to study inhomogeneities seen in the individual CO first overtone lines. Our AMBER observations represent the highest spatial resolution achieved for Betelgeuse, corresponding to five resolution elements over its stellar disk. The AMBER visibilities and closure phases in the $K$-band continuum can be reasonably fitted by a uniform disk with a diameter of $43.19 \pm 0.03$ mas or a limb-darkening disk with $43.56 \pm 0.06$ mas and a limb-darkening parameter of $(1.2 \pm 0.07) \times 10^{-1}$. On the other hand, our AMBER data in the CO lines reveal salient inhomogeneous structures. The visibilities and phases (closure phases, as well as differential phases representing asymmetry in lines with respect to the continuum) measured within the $\mathrm{CO}$ lines show that the blue and red wings originate in spatially distinct regions over the stellar disk, indicating an inhomogeneous velocity field that makes the star appear different in the blue and red wings. Our AMBER data in the CO lines can be roughly explained by a simple model, in which a patch of $\mathrm{CO}$ gas is moving outward or inward with velocities of $10-15 \mathrm{~km} \mathrm{~s}^{-1}$, while the $\mathrm{CO}$ gas in the remaining region in the atmosphere is moving in the opposite direction at the same velocities. These velocities compare favorably with the macroturbulent velocities of $10-20 \mathrm{~km} \mathrm{~s}^{-1}$ derived by spectroscopic analyses. Also, the AMBER data are consistent with the presence of warm molecular layers (so-called MOLsphere) extending to $\sim 1.4-1.5 R_{\star}$ with a CO column density of $\sim 1 \times 10^{20} \mathrm{~cm}^{-2}$. However, the present data are insufficient to constrain the surface pattern uniquely or to reconstruct an image. Our AMBER observations of Betelgeuse are the first spatially resolved study of the macroturbulence in a stellar atmosphere (photosphere and possibly MOLsphere as well) other than the Sun. The spatially resolved CO gas motion is likely to be related to convective motion in the upper atmosphere or intermittent mass ejections in clumps or arcs.

\section{References}

Ohnaka, K. et al. 2009, A\&A in press, astro-ph/0906.4729. 